\title{
Affective stimulus properties influence size perception and the Ebbinghaus illusion
}

\author{
Niek R. van Ulzen • Gün R. Semin • \\ Raôul R. D. Oudejans · Peter J. Beek
}

Received: 2 October 2006 / Accepted: 27 February 2007 / Published online: 5 April 2007

(C) Springer-Verlag 2007

\begin{abstract}
In the New Look literature of the 1950s, it has been suggested that size judgments are dependent on the affective content of stimuli. This suggestion, however, has been 'discredited' due to contradictory findings and methodological problems. In the present study, we revisited this forgotten issue in two experiments. The first experiment investigated the influence of affective content on size perception by examining judgments of the size of target circles with and without affectively loaded (i.e., positive, neutral, and negative) pictures. Circles with a picture were estimated to be smaller than circles without a picture, and circles with a negative picture were estimated to be larger than circles with a positive or a neutral picture confirming the suggestion from the 1950s that size perception is influenced by affective content, an effect notably confined to negatively loaded stimuli. In a second experiment, we examined whether affective content influenced the Ebbinghaus illusion. Participants judged the size of a target circle whereby target and flanker circles differed in affective loading. The results replicated the first experiment. Additionally, the Ebbinghaus illusion was shown to be weakest for a negatively loaded target with positively loaded and blank flankers. A plausible explanation for both sets of experimental findings is that negatively loaded stimuli are more attention demanding than positively loaded or neutral stimuli.
\end{abstract}

\footnotetext{
N. R. van Ulzen $(\varangle) \cdot$ G. R. Semin · R. R. D. Oudejans · P. J. Beek Department of Social Psychology, Vrije Universiteit,

Van der Boechorststraat 1, 1081 BT, Amsterdam,

The Netherlands

e-mail: NR.van.Ulzen@psy.vu.nl
}

\section{Introduction}

According to the New Look movement that figured prominently in the 1950s, perceptual processes such as size judgments are influenced by affectively loaded or 'value' variables. For example, Bruner and Goodman (1947) observed that coins were perceived as larger than same-size cardboard discs, and more so by poor children than by rich children. Why precisely participants overestimated the size of the coins was not fully clear, but it was conjectured that, because coin size is typically positively correlated with value, coins of a given size were seen as larger than their non-valuable counterparts (Smith, Fuller, \& Forrest, 1975; Taifel, 1957). Another experiment by Bruner and Postman (1948) used positive (dollar sign), neutral (a square) and negative (swastika) symbols projected on plastic discs of varying size. An overestimation was found for stimuli containing a symbol indicating something positive or negative. It was argued that what is salient to a person looms larger in his or her perception. Subsequent studies, however, yielded inconsistent results regarding the effect of value on size judgments (see Jenkin, 1957; Tajfel, 1957). For example, Klein, Schlesinger and Meister (1951) tried to replicate the findings of Bruner and Postman (1948) but merely found a slight tendency towards overestimation of discs containing positive or negative symbols compared to neutral discs. According to Klein et al. (1951), distortions in size perception are more dependent on the method used for size judgments than on the value of the stimuli. As a consequence of empirical inconsistencies and methodological shortcomings (Tajfel, 1957), the suggestion that size judgments are dependent on the affective content of stimuli fell into discredit. However, given all results obtained in this field of inquiry it cannot be excluded altogether that affective content contributes to size perception (Jenkin, 1957). With the 
advent of the notion of embodied cognition, it has become evident that the human eye is not solely an organ for vision, but that visual perception is permeated by biological needs, emotional states, and action intentions, which in turn depend on the prevailing context (Proffitt, 2006; Proffitt, Stefanucci, Banton, \& Epstein, 2003). In view of this development, we considered it worthwhile to reexamine the influence of affective content on size perception.

To this end, we conducted two experiments, one addressing the topic of interest in a plain, straightforward manner, and the other examining whether affective and contextual properties interact when judging size. This second experiment was motivated from the consideration that affect may influence the salience of contextual stimuli differently than that of focal stimuli. This consideration was prompted, on the one hand, by studies on the influence of affective content on visual attention, and on the other hand by studies on the influence of cognitive and social factors on the Ebbinghaus illusion. In the former research, it was found that affective content influences the salience of stimuli in that negative targets in a positive or neutral context stand out more than positive targets in a negative or neutral context (e.g., Hansen \& Hansen, 1988; Öhman, Lundqvist, $\&$ Esteves, 2001). In the latter research, it has been found that both judgmental comparisons and attentional processes play into the Ebbinghaus illusion (Coren \& Enns, 1993; Shulman, 1992; Stapel \& Koomen, 1997). Coren and Enns (1993) showed that when target and flanker circles are drawn from the same conceptual category (e.g., a dog surrounded by other dogs, a female face surrounded by other female faces) the Ebbinghaus illusion is stronger than for conceptually unrelated objects (e.g., a dog surrounded by shoes). Stapel and Koomen (1997) replicated this finding for social categories. Participants were provided with social knowledge of target and context stimuli without clear physical cues. The Ebbinghaus illusion was stronger for socially similar stimuli (lawyers) than when target and context belonged to different social categories (student vs. lawyers). Importantly, in a study by Muise, Brun and Porelle (1997) children judged the size of an 'Oreo cookie' as larger than a normal target circle of the same size, form and color when surrounded by large flanker circles, providing some indication that the value of the target may influence the Ebbinghaus illusion.

\section{Experiment 1: valence and judgments of circle size}

Experiment 1 was designed to examine how affective content influences size judgments. Previous studies on perceptual distortions in coin size judgments or judgments of objects with a certain emotional value (e.g., Bruner \& Goodman, 1947; Bruner \& Postman, 1948; Klein et al.,
1951) produced ambiguous results and suffered from methodological shortcomings. We reexamined the issue using controlled manipulations of valence and precise, computerized measurements of size judgments. In addition, blank circles were used to assess the influence of optical structure itself.

Method

\section{Participants}

Sixty-two Dutch students (mean age $=20$ years, range $=$ 17-28 years) recruited from the VU University Amsterdam participated in this experiment on a paid voluntary basis. Participants were assigned randomly to conditions.

\section{Materials}

Three positive, three neutral and three negative pictures were selected from the International Affective Picture System (IAPS) database (Center for the Study of Emotion and Attention, 1995; Lang, Bradley, \& Cuthbert, 2001) based on their valence, arousal and dominance norms (see "Appendix"). The pictures were portrayed as circles on a computer screen, using a blank circle as control stimulus. Target circles had the same diameter $(49.5 \mathrm{~mm})$, but the size of the object on the picture was varied so that for each picture, a normal (small) and a magnified (large) version was available. The diameter of the target circles used in the catch trials (see below) was varied as well to prevent that participants would use undesired cognitive strategies like moving the mouse over a fixed distance.

\section{Procedure}

Participants were seated in cubicles equipped with a computer. Instructions and stimuli were presented on a 19 in. computer screen. Participants were instructed to match the size of a comparison circle with the size of the target circle. The comparison circle was either smaller or larger than the target circle and could be enlarged or reduced in size to match the size of the target circle by pushing or pulling the computer's mouse. ${ }^{1}$ This variation was counterbalanced across participants. Participants initiated a trial by pressing a key. After a variable duration (1,000-1,500 ms) the target and comparison

\footnotetext{
${ }^{1}$ It is known that judgments are influenced by direction (e.g., Pufall \& Dunbar, 1992). Participants tend to overestimate when the comparison circle is larger than the target circle and has to be reduced in size, and underestimate when the comparison circle is smaller than the target circle and has to be enlarged. To also control for possible effects of movement direction we included movement of the mouse, pushing away from or pulling towards you, as an additional variable.
} 
circle appeared simultaneously on the screen. The target circle appeared in the middle of the screen; the comparison circle appeared in the lower right corner of the screen. Participants then adjusted the size of the comparison circle to match the perceived size of the target circle. If, in their judgment, this was the case, participants pressed another key where after the screen turned blank. After $2 \mathrm{~s}$ the next trial started and the procedure was repeated.

\section{Data analysis}

Results were analyzed using a $2 \times 2 \times 2 \times 4$ mixed design ANOVA with one between-participants variable, i.e., Group (enlarge vs. reduce), and three within-participants variables, i.e., Mouse Movement (push and pull), Picture Size (small and large), and Valence (positive, neutral, negative, and blank). Participants performed 60 trials in total, 48 (3 pictures per valence) of which were used for further statistical analysis. The remaining 12 trials were catch trials, and comprised 6 trials in which the target circle was larger $(52 \mathrm{~mm})$ and 6 trials in which the target circle was smaller $(47 \mathrm{~mm})$ than the target circle used in the experimental trials. The pictures portrayed on these catch circles were chosen from the sets of positive, neutral and negative IAPS pictures as used in the experimental trials (see "Appendix"). Significance level was set at $P<0.05$, and partial eta squared $\left(\eta_{\mathrm{p}}^{2}\right)$ and $r_{\text {contrast }}$ (see Rosenthal, Rosnow, \& Rubin, 2000) were used as measures of effect size.

\section{Results}

Twelve participants whose judgments deviated from the mean by more than three standard deviations were excluded from further analyses leaving a total of 50 participants (25 in each group). For these participants, valence had a significant effect on size judgments, $F(2.19$, $105.27)=47.37, P<0.0001, \eta_{\mathrm{p}}^{2}=0.50$ (see Fig. 1 ). ${ }^{2}$ Posthoc contrast analyses showed that the circles with pictures were significantly underestimated compared to the blank circles, $F(1,48)=75.06, P<0.0001, r_{\text {contrast }}=0.78$, and that circles with negative pictures were significantly less underestimated than circles with positive, $F(1,48)=5.08$, $P<0.05, \quad r_{\text {contrast }}=0.31$, and neutral pictures, $F(1$, $48)=6.46, P<0.05, r_{\text {contrast }}=0.34$. The influence of picture size on size judgments was not significant. Although the Picture Size $\times$ Valence interaction was significant, $F(3,144)=3.39, P<0.05, \eta_{\mathrm{p}}^{2}=0.07$, this effect was solely due to a significant difference between small and large

\footnotetext{
${ }^{2}$ Degrees of freedom were adjusted using a Greenhouse-Geisser adjustment when the data did not meet the sphericity assumption.
}

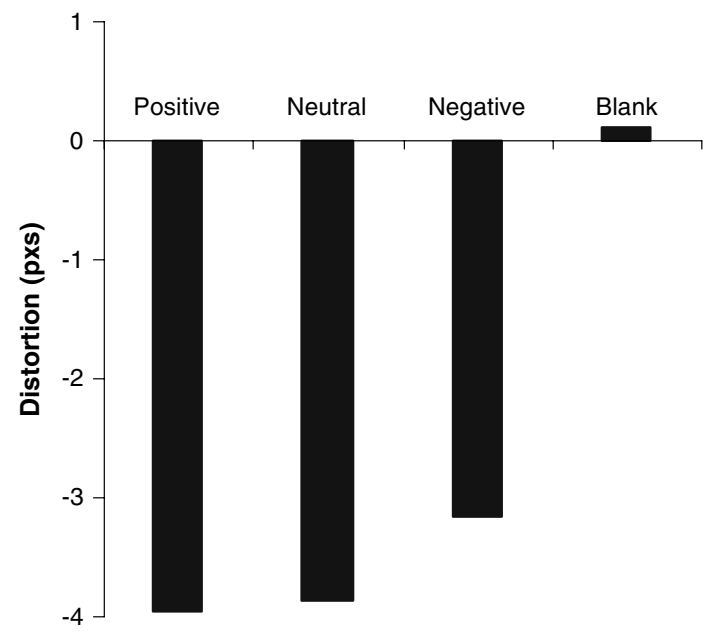

Fig. 1 Size judgments as a function of circle content. Zero represents the actual size of the circle; the apparent circle size is given by the distortion from zero, negative signs indicate underestimation, positive signs indicate overestimation

neutral pictures, ${ }^{3} F(1,48)=8.154, P<0.01, r_{\text {contrast }}=$ 0.38. To make sure that the observed effect of affective content on size perception could not be ascribed to a difference in the brightness of the stimuli, we tested the grey tones of the positively loaded stimuli to that of the negatively loaded stimuli. As this test revealed no significant difference we concluded that the observed effect could indeed be ascribed to a difference in valence.

\section{Discussion}

Our results confirmed the suggestion from the 1950s that size perception is dependent on affective content in that negatively loaded circles were underestimated less than neutral and positively loaded circles. For a full appreciation of the results, it is important to realize that all circles with a picture, were underestimated compared to the blank circles, implying that, apparently, size judgment is also dependent on the optical structure of the stimuli (i.e., the presence or absence of a picture), regardless of its affective or semantic content. Previous studies on visual illusions proposed that contour effects may give rise to perceptual distortions in that nearby contours attract the contours of a target, making it look larger, whereas more distant contours repel the contours of a target, making it look smaller (e.g., Badcock \& Westheimer, 1985; Bondarko \& Danilova, 1999; Jaeger,

\footnotetext{
${ }^{3}$ In all experiments, the variables Group and Mouse Movement were included to control for possible confounding effects. These factors caused significant interactions, which reflected a different pattern of responses for the neutral pictures not relevant for the current discussion. We refrain from reporting such significant interactions unless they contribute to an understanding of how task-related factors mediate the influence of affect on perception.
} 
1978). It could be the case that the optical contours of the picture on the circle 'attract' the contours of the circle, making it look smaller. This argument is also in line with Klein and colleagues' (1951) notion that the pictorial properties of the value symbols might have been an important factor overlooked by Bruner and Postman (1948). In the present experiment, the influence of affect on size perception was more subtle than the effect of optical structure as such, but statistically significant nevertheless. After all, the negatively loaded stimuli were underestimated less, and thus were perceived as larger, than the neutral and positively loaded stimuli (for which the size judgments were similar). This is in accordance with Bruner and Postman's (1948) proposal that what is salient to a person looms larger in his or her perception.

\section{Experiment 2: the Ebbinghaus illusion with valence-incongruent flanker-target combinations}

As argued in the "Introduction", affective content may influence the Ebbinghaus illusion when the valence of the target is different from that of the flanker. After all, when a target is placed in an incongruent affective context the relative salience of target and flanker may have a bearing on target size evaluations, because a negative target in a positive or neutral context is known to stand out more and to lead to faster reaction times than a positive target in a negative or neutral context (e.g., Hansen \& Hansen, 1988; Öhman, Flykt, \& Esteves, 2001; Öhman, Lundqvist, \& Esteves, 2001). If so, the Ebbinghaus illusion would be attenuated with a negative target and positive (or blank) flankers and would be enhanced with a positive target and negative (or blank) flankers. Experiment 2 was designed to test this prediction.

\section{Method}

\section{Participants}

Seventy-four Dutch students (mean age $=21$ years, range $=18-29$ years) from the VU University Amsterdam participated in this experiment on a paid voluntary basis. They were assigned randomly over conditions.

\section{Materials}

The stimuli consisted of target circles embedded in two different contextual displays, one with eleven small flanker circles $(16.5 \mathrm{~mm}$ in diameter) and one with five large flanker circles $(95.7 \mathrm{~mm}$ in diameter). The size of the flankers was varied to create two instantiations of the Ebbinghaus illusion. For all displays, the target circle had a diameter of $49.5 \mathrm{~mm}$, except in the catch trials (see below) where the diameter of the target circles was varied. The affective content of the display was manipulated by projecting IAPS pictures on the target and flanker circles. The valence of the target circle always differed from that of the flanker circles. Seven flanker-target configurations were used: positive targets surrounded by negative (1, TposFneg) or blank (2, TposFbla) flankers; negative targets surrounded by positive (3, TnegFpos) or blank (4, TnegFbla) flankers; and blank targets surrounded by positive (5, TblaFpos), negative (6, TblaFneg) or blank (7, TblaFbla) flankers. The experimental predictions directly pertained to conditions 1 and 3 and conditions 2 and 4, i.e., the conditions in which the target was either positively or negatively loaded, while the flankers were either incongruently loaded or blank. Conditions 5 and 6 were included to determine whether positively loaded flankers influenced the illusion differently than negatively loaded flankers in the case of a blank target. The last configuration with a blank target surrounded by blank flankers served as control condition. For the two valences (i.e., positive and negative) three pictures were chosen from the IAPS database (see "Appendix"). For the negative-positive and positive-negative flanker-target configurations valenced pictures were assigned randomly (from a pool of nine possible flanker-target combinations) to the target and flanker circles.

\section{Procedure}

The procedure was identical to that used in Experiment 1.

\section{Data analysis}

Participants performed 50 trials in total, 42 (3 repetitions per flanker-target combination) of which were used for further statistical analysis. The remaining 8 trials were included as catch trials, and comprised 4 trials in which the target circle was larger $(52 \mathrm{~mm})$ and 4 trials in which the target circle was smaller $(47 \mathrm{~mm})$ than the target circle used in the experimental trials.

The results in terms of the difference in the strength of the illusion as invoked by the size manipulation of the flankers were compared across target-flanker configurations 1 through 4 using planned comparisons. In addition, a planned comparison was conducted for target-flanker configurations 5 and 6 , again with regard to the strength of the illusion. Finally, two planned comparisons were performed to verify the results of Experiment 1, namely, the effect of valence on size perception as such and the effect of optical structure on size perception as such. This was done by testing the results for all negatively loaded targets against all positively loaded targets and by testing all targets with a picture against all blank targets. Significance level was set 


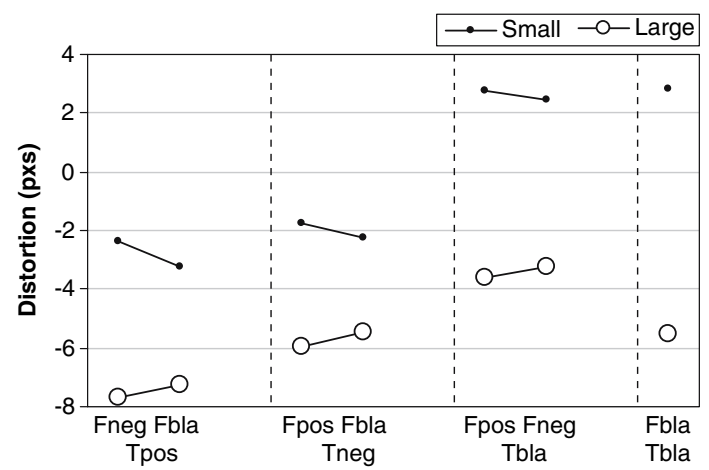

Fig. 2 Size judgments as a function of flanker size (small vs. large) and target-flanker configuration. Positive targets (Tpos) were surrounded by negative (Fneg) or blank (Fbla) flankers, negative targets (Tneg) by positive (Fpos) or blank flankers, and blank targets (Tbla) by positive, negative or blank flankers

at $P<0.05$, and $r_{\text {contrast }}$ (see Rosenthal, Rosnow, \& Rubin, 2000) was used as a measure of effect size.

\section{Results}

Eight participants were excluded from the analyses because their judgments deviated more than 3 standard deviations from the mean, leaving 66 participants for further analyses. The main results are depicted in Fig. 2, and suggest that the size manipulation of the flankers had a greater effect for the positively loaded targets than for the negatively loaded targets, regardless of whether the flankers were affectively loaded or blank. The planned comparison in question revealed that this difference tended toward significance, $F(1,64)=3.84, P=0.054, r_{\text {contrast }}=0.24$. When this analysis was restricted to participants who were susceptible to the illusion $(n=63)$, it was significant, $F(1,61)=5.37$, $P=0.024, r_{\text {contrast }}=0.28$. If it was conducted for the incongruently loaded and blank flankers separately, the observed tendency turned into a non-significant effect $(P>0.1)$. The planned comparison of the effect of the size manipulation across conditions 5 and 6 was not significant. Planned comparisons further revealed that positively loaded targets were judged as significantly smaller than negatively loaded targets, $F(1,64)=18.81, P<0.0001, r_{\text {contrast }}=0.48$, regardless of whether the flankers were affectively loaded or blank, and that targets with a picture were judged as significantly smaller than blank targets, $F(1,64)=117.57$, $P<0.0001, r_{\text {contrast }}=0.80$, again regardless of the nature of the flankers. As in Experiment 1, we ascertained that the reported effects of affective content on size perception could not be ascribed to a difference in the brightness of the stimuli by testing the grey tones of the positively loaded stimuli to that of the negatively loaded stimuli. Again, this test turned out non-significant.

\section{Discussion}

In line with the results of Experiment 1, targets with a picture were underestimated compared to blank targets with negatively loaded targets being underestimated less than positively loaded targets. The critical question of Experiment 2 was whether the Ebbinghaus illusion was modulated in any way by affective content, in particular in the case of incongruent valences of target and flankers. Planned comparisons revealed that such modulation is indeed the case. If a positively loaded target was surrounded by negatively loaded or blank flankers, then the variation induced in the Ebbinghaus illusion by changing flanker size was greater than for a negatively loaded target surrounded by positively loaded or blank flankers. As the nature of the flankers (i.e., affectively loaded versus blank) was of little consequence, it may be concluded that the critical difference between the two configurations pertained to the valence of the target. It appears that if the target was negatively loaded, it attracted more attention than when it was positively loaded, at the expense of the attention paid to the flankers. As a consequence, the Ebbinghaus illusion was reduced for negatively loaded targets.

\section{General discussion}

The reported studies examined the influence of affect (valence) on size perception as such and on size perception in the context of the Ebbinghaus illusion. In Experiment 1, we found that the size of a circular object is underestimated when the object is optically structured compared to when it is blank. In addition, we found that this underestimation was less when the object contained a negatively loaded picture than when it contained a positively loaded or neutral picture. Both findings were replicated in Experiment 2, which was conducted to examine whether the influence of affect (valence) on size perception was modulated by contextual stimuli, using the Ebbinghaus illusion as experimental paradigm. We found a clear indication for an influence of affective content on the Ebbinghaus illusion in an analysis including all participants. This effect was stronger (i.e., significant) when three participants who were not susceptible to the Ebbinghaus illusion were excluded from the analyses.

Overall, it appears that the results of the present studies can be accounted for in terms of three principles: (1) Optical structure leads to underestimations of target size, presumably because the contours of the optical structure 'attract' the contours of the circle, making it look smaller (e.g., Badcock \& Westheimer, 1985; Bondarko \& Danilova, 1999; Jaeger, 1978); (2) Stimuli that are motivationally salient, namely, negative stimuli, are underestimated less 
compared to positive and neutral stimuli, possibly because negatively loaded pictures loom larger than positively loaded and neutral pictures (Bruner \& Postman, 1948); and (3) In a similar vein, because negatively loaded targets are more attention demanding than positively loaded and neutral (i.e., blank) targets (e.g., Fox, Russo, \& Dutton, 2002; Georgiou et al., 2005), the Ebbinghaus illusion is weaker for the former than for the latter targets.

Those results are of interest for (at least) two reasons. First and foremost, they resurrect the findings and insights of the New Look psychology, which were perhaps dismissed too quickly. After all, even though it was the case that results were mixed and experiments suffered from methodological imperfections, the insight gained at the time may still be valid, as indeed is suggested by the present results. Second, they fuel the notion of embodied cognition by demonstrating that a basic visual phenomenon like the Ebbinghaus illusion is modulated by the affective content of the stimuli. Whereas some authors, like Coren and Enns (1993) and Stapel and Koomen (1997), have demonstrated that the strength of the Ebbinghaus illusion is codependent on cognitive operations, it is shown here that also the affective content of the stimuli may play into this illusion, particularly when the targets are negatively loaded.

Acknowledgments This study was supported by the Royal Netherlands Academy of Arts and Sciences (ISK/4583/PAH) and NWO (Netherlands Organization for Scientific research), Research Grant 051.02.090. The authors wish to thank Eric van Rossum for his technical support in preparing the stimuli.

\section{Appendix}

Table 1
Table 1 IAPS pictures as used in experiments 1 and 2

\begin{tabular}{lllllll}
\hline Experiment & IAPS\# & Positive & IAPS\# & Negative & IAPS\# & Neutral \\
\hline 1 & 1463 & Kittens & 6260 & Aimed gun & 1616 & Bird \\
& 1750 & Bunnies & 9301 & Toilet & 5500 & Mushrooms \\
& 5010 & Flower & 9440 & Skulls & 7009 & Mug \\
2 & 1463 & Kittens & 1201 & Spider & & \\
& 5001 & Sunflower & 6260 & Aimed gun & & \\
& 7260 & Torte & 9440 & Skulls & & \\
\hline
\end{tabular}

\section{References}

Badcock, D. R., \& Westheimer, G. (1985). Spatial location and hyperacuity-the center-surround localization contribution function has 2 substrates. Vision Research, 25, 1259-1267.

Bondarko, V. M., \& Danilova, M. V. (1999). Spatial interval discrimination in the presence of flanking lines. Spatial Vision, 12, 239253

Bruner, J. S., \& Goodman, C. C. (1947). Value and need as organizing factors in perception. Journal of Abnormal and Social Psychology, 42, 33-44.

Bruner, J. S., \& Postman, L. (1948). Symbolic value as an organizing factor in perception. Journal of Social Psychology, 27, 203-208.

Center for the Study of Emotion and Attention (CSEA-NIMH) (1995). The international affective picture system: Digitized photographs. Gainsville: University of Florida, Center for Research in Psychophysiology.

Coren, S., \& Enns, J. T. (1993). Size contrast as a function of conceptual similarity between test and inducers. Perception and Psychophysics, 54, 579-588.

Fox, E., Russo, R., \& Dutton, K. (2002). Attentional bias for threat: Evidence for delayed disengagement from emotional faces. Cognition and Emotion, 16, 355-379.

Georgiou, G. A., Bleakley, C., Hayward, J., Russo, R., Dutton, K., Eltiti, S., et al. (2005). Focusing on fear: Attentional disengagement from emotional faces. Visual Cognition, 12, 145-158.
Hansen, C. H., \& Hansen, R. D. (1988). Finding the face in the crowd - an anger superiority effect. Journal of Personality and Social Psychology, 54, 917-924.

Jaeger, T. (1978). Ebbinghaus illusions-size contrast or contour interaction phenomena. Perception and Psychophysics, 24, $337-$ 342.

Jenkin, N. (1957). Affective processes in perception. Psychological Bulletin, 54, 100-127.

Klein, G. S., Schlesinger, H. J., \& Meister, D. E. (1951). The effect of personal values on perception-an experimental critique. Psychological Review, 58, 96-112.

Lang, P. J., Bradley, M. M., \& Cuthbert, B. N. (2001). International affective picture system (IAPS): Instruction manual and affective ratings. Technical report A-5. The Center for Research in Psychophysiology, University of Florida.

Muise, J. G., Brun, V., \& Porelle, M. (1997). Salience of central figure in the Ebbinghaus illusion: The Oreo cookie effect. Perceptual and Motor Skills, 85, 1203-1208.

Öhman, A., Flykt, A., \& Esteves, F. (2001). Emotion drives attention: Detecting the snake in the grass. Journal of Experimental Psychology: General, 130, 466-478.

Öhman, A., Lundqvist, D., \& Esteves, F. (2001). The face in the crowd revisited: A threat advantage with schematic stimuli. Journal of Personality and Social Psychology, 80, 381-396.

Proffitt, D. R. (2006). Embodied perception and the economy of action. Perspectives on Psychological Science, 1, 110-122. 
Proffitt, D. R., Stefanucci, J., Banton, T., \& Epstein, W. (2003). The role of effort in distance perception. Psychological Science, 14, 106-113.

Pufall, P. B., \& Dunbar, C. (1992). Perceiving whether or not the world affords stepping onto and over: A developmental study. Ecological Psychology, 4, 17-38.

Rosenthal, R., Rosnow, R. L., \& Rubin, D. B. (2000). Contrast and effect sizes in behavioral research. New York: Cambridge University Press

Shulman, G. L. (1992). Attentional modulation of size contrast. Quarterly Journal of Experimental Psychology Section A-Human Experimental Psychology, 45, 529-546.
Smith, H. V., Fuller, R. G. C., \& Forrest, D. W. (1975). Coin value and perceived size: a longitudinal study. Perceptual and Motor Skills, 41, 227-232.

Stapel, D. A., \& Koomen, W. (1997). Social categorization and perceptual judgment of size: When perception is social. Journal of Personality and Social Psychology, 73, 1177-1190.

Tajfel, H. (1957). Value and the perceptual judgment of magnitude. Psychological Review, 64, 192-204. 